\title{
GROWTH AND DECAY ESTIMATES NEAR NON-ELEMENTARY STATIONARY POINTS
}

\author{
COURTNEY COLEMAN
}

1. Introduction. Local growth and decay estimates near the stationary point at the origin are derived in $\S 3$ for solutions of the vector system,

$$
\begin{aligned}
& d x / d t \equiv \dot{x}=A(x)+f^{*}(x, y), \\
& d y / d t \equiv \dot{y}=B(y)+g^{*}(x, y),
\end{aligned}
$$

where $A(x)$ and $B(y)$ are homogeneous of degree $m>1$ in the components of $x$ and $y$, respectively, and $f^{*}$ and $g^{*}$ are of order greater than $m$ in $\|(x, y)\|$ near the origin. It is assumed that $x=0$ is asymptotically stable and $y=0$ is asymptotically unstable for the homogeneous systems of first approximation,

$$
\begin{aligned}
& \text { (a) } \dot{x}=A(x), \\
& \text { (b) } \dot{y}=B(y) .
\end{aligned}
$$

In order to derive the estimates in $\S 3$, various results are needed concerning solutions of a homogeneous system such as (2)(a). These are derived in $\$ 2$ and are based on work of Hahn $[\mathbf{4} ; \mathbf{5}]$, Lefschetz $[\mathbf{8}]$, and Zubov $[\mathbf{1 2}]$. A fundamental question, given a homogeneous system such as

$$
\dot{z}=C(z), \quad \operatorname{dim} z=\operatorname{dim} x+\operatorname{dim} y,
$$

is whether there exists a linear variable change, $(x, y)=T z$, which uncouples (3), transforming it into (2). Extending an idea of Markus [9], the existence of such a $T$ is tied in with a decomposition problem for a certain non-associative algebra. This is pursued in $\S 4$. Where such a reduction of (3) is possible, we then have a corresponding reduction of a system such as

$$
\dot{z}=C(z)+\text { higher order terms }
$$

to system (1).

The results of this paper can be compared with the known exponential estimates for solutions of (1) when $m=1$ and $A(x)=A x, B(y)=B y$, where $A$ and $B$ are matrices, the eigenvalues of $A$ having negative real parts, those of $B$ positive real parts; see e.g., $[2 ; \mathbf{4} ; \mathbf{6}]$. In this case, Grobman $[3]$ and Hartman [6, Chapter IX; 7] have used these exponential estimates to solve the topological equivalence problem for (1) at the origin; i.e., they showed that there is a homeomorphism of a neighbourhood of the origin in to itself mapping solutions of (1) onto solutions of (2). Reiziňš [10] has extended

Received February 2, 1970. This research was supported in part by the National Science Foundation under grant number GP-12407. 
this to the case when one of the eigenvalues of $A$ vanishes. In a subsequent paper the estimates derived in $\S 3$ will be used to solve the topological equivalence problem for (1) at the origin when $m>1$.

2. Decay estimates for an asymptotically stable homogeneous system. Let $A(x)$ be a $k$-vector whose components are homogeneous functions of degree $m>1$ in the components of the $k$-vector $x$. Let the critical point at the origin of the system

$$
d x / d t=A(x)
$$

be asymptotically stable. We are interested in finding upper and lower bounds on $\left|x\left(t, x_{0}\right)\right|, t \geqq 0$, where $x\left(t, x_{0}\right)$ is the solution of $(5)$ for which $x\left(0, x_{0}\right)=x_{0}$.

First we shall state a number of easily proved properties for homogeneous systems such as (5); see [12].

(a) If $c>0$, then $c x\left(c^{m-1} t, x_{0}\right)=x\left(t, c x_{0}\right)$; this is true whether or not the origin is asymptotically stable.

(b) The asymptotic stability of (5) at the origin is global.

(c) If $m$ is an integer, then the asymptotic stability implies that $m$ is odd.

THEOREM 1. There exist positive constants $a, b, c, T$, such that

$$
a t^{1 /(1-m)} \leqq\left|x\left(t, x_{0}\right)\right| \leqq b t^{1 /(1-m)}
$$

for all $t>T\left|x_{0}\right|^{1 /(1-m)}$; another lower bound is given by $\left|x_{0}\right|\left[1+c\left|x_{0}\right|^{m-1} t\right]^{1 /(1-m)}$ valid for all $t \geqq 0$.

Note that the existence of the upper bound implies that the origin of (5) is asymptotically stable.

Proof. $B(\rho)$ will denote the open ball of radius $\rho$ centred at the origin of $E^{n}, S(\rho)$ its boundary. Let $\rho_{1}>\rho_{2}>0$ and let $t^{*}\left(\rho_{1}, \rho_{2}, x_{0}\right)$ be the least positive value of $t$ for which $x\left(t, x_{0}\right) \in S\left(\rho_{2}\right)$ if $x_{0} \in S\left(\rho_{1}\right)$. The existence of $t^{*}$ follows from the asymptotic stability (global because of the homogeneity of $A$ ) of the origin. For the same reason there exists $T^{*}\left(\rho_{1}, \rho_{2}, x_{0}\right)$, the least value of $t$ for which $x\left(t, x_{0}\right) \in B\left(\rho_{2}\right)$ for all $t>T^{*}, x_{0} \in S\left(\rho_{1}\right)$. It will now be shown that the infimum of the $t^{*} \mathrm{~s}$ and the supremum of the $T^{*} \mathrm{~s}$ over $S\left(\rho_{1}\right)$ (for fixed $\rho_{1}$ and $\rho_{2}$ ) both exist and are positive.

Let $\alpha=\inf (y \cdot A y)$ over $S(1)$ where - denotes the inner product. $\alpha$ is negative. For, if $\alpha \geqq 0$, then $d r / d t=r^{m}(y \cdot A(y)) \geqq 0$ (here, $r=|x|$ and $|y|=1)$ and $|x|$ could not tend to 0 as $t$ tends to infinity, contradicting the asymptotic stability of the origin. Comparing the above differential equation with the equation $d r / d t=r^{m}$, it can be seen that

$$
0<\frac{\rho_{1}{ }^{m-1}-\rho_{2}{ }^{m-1}}{\left(\rho_{1} \rho_{2}\right)^{m-1} \alpha(m-1)} \leqq t^{*}\left(\rho_{1}, \rho_{2}, x_{0}\right) .
$$

This is shown by finding the value of $t$ for which the solution of $d r / d t=\alpha r^{m}$ beginning at $\rho_{1}$ for $t=0$ reaches $r=\rho_{2}$. Thus, the $t^{*}$ s have an infimum $t^{*}\left(\rho_{1}, \rho_{2}\right)$ over $S\left(\rho_{1}\right)$. 
For later use in the proof, some estimates involving $t^{*}, \rho_{1}$, and $\rho_{2}$ are now derived. If it takes an orbit $x\left(t, x_{0}\right)$ time $\tau$ to get from $S\left(\rho_{1}\right)$ to $S\left(\rho_{2}\right)$, it will take the orbit $x\left(t, c x_{0}\right), c>0$, time $c^{1-m} \tau$ to get from $S\left(c \rho_{1}\right)$ to $S\left(c \rho_{2}\right)$. Hence, $t^{*}\left(2^{-k} \rho_{1}, 2^{-k-1} \rho_{1}\right)=2^{k(m-1)} t^{*}\left(\rho_{1}, \frac{1}{2} \rho_{1}\right)$. Using this and the fact that $t^{*}\left(\rho_{1}, \rho_{3}\right) \geqq$ $t^{*}\left(\rho_{1}, \rho_{2}\right)+t^{*}\left(\rho_{2}, \rho_{3}\right)$ if $\rho_{1}>\rho_{2}>\rho_{3}>0$, it is seen that

$$
t^{*}\left(\rho_{1}, 2^{-k-1} \rho_{1}\right) \geqq t^{*}\left(\rho_{1}, 2^{-1} \rho_{1}\right)\left[1+2^{m-1}+\ldots+2^{k m-k}\right] .
$$

Using the inequality derived in the paragraph above and this last inequality, we obtain

$$
t^{*}=t^{*}\left(\rho_{1}, 2^{-k-1} \rho_{1}, x_{0}\right) \geqq t^{*}\left(\rho_{1}, 2^{-k-1} \rho_{1}\right) \geqq \frac{\left(2^{m-1}-1\right) 2^{k(m-1)} C(k)}{\rho_{1}{ }^{m-1} \alpha(m-1)},
$$

where $C(k)$ depends only upon $k$ and lies between 1 and 2. From this follows the estimate

$$
a\left(t^{*}\right)^{1 /(1-m)} \leqq 2^{-k} \rho_{1},
$$

where $a$ is a positive constant independent of $\rho_{1}$ and $k$.

The $T^{*}$ s must now be shown to have a supremum over $S\left(\rho_{1}\right)$ for fixed $\rho_{1}$ and $\rho_{2}$. Let $x_{0} \in S\left(\rho_{1}\right)$ and let $N\left(x_{0}\right)$ be a neighbourhood (relative to $S\left(\rho_{1}\right)$ ) of $x_{0}$ such that $\left\{T^{*}\left(\rho_{1}, \rho_{2}, x\right): x \in N\left(x_{0}\right)\right\}$ is bounded from above. Suppose that for some $x_{0} \in S\left(\rho_{1}\right)$ the desired $N\left(x_{0}\right)$ does not exist. There exists a sequence $\left(t_{n}, x_{n}\right), x_{n} \rightarrow x_{0}, x_{n} \in S\left(\rho_{1}\right), t_{n} \rightarrow \infty$, such that $x\left(t_{n}, x_{n}\right) \notin B\left(\rho_{2}\right)$. Because of the asymptotic stability there is a $\rho_{3}, \frac{1}{2} \rho_{2}>\rho_{3}>0$, such that any orbit in $B\left(\rho_{3}\right)$ for some $t$ remains in $B\left(\frac{1}{2} \rho_{2}\right)$ for all larger $t$. A contradiction will arise once it is known that for some $n$ and $t, 0 \leqq t \leqq t_{n}, x\left(t, x_{n}\right) \in B\left(\rho_{3}\right)$. Let $k$ be so large that $2^{-k} \rho_{1}>\frac{1}{2} \rho_{3}$. Then for all $t, t \geqq T^{*}\left(\rho_{1}, 2^{-k} \rho_{1}, x_{0}\right)$, $x\left(t, x_{0}\right) \in B\left(\frac{1}{2} \rho_{3}\right)$. Since $x\left(t, x_{0}\right)$ is continuous in $t$ and $x_{0}$, there is an $n_{0}$ such that for all $t, 0 \leqq t \leqq T^{*}\left(\rho_{1}, 2^{-k} \rho_{1}, x_{0}\right)$, and all $n>n_{0},\left|x\left(t, x_{0}\right)-x\left(t, x_{n}\right)\right|<\frac{1}{2} \rho_{3}$. For such $n, x\left(T^{*}\left(\rho_{1}, 2^{-k} \rho_{1}, x_{n}\right), x_{n}\right) \in B\left(\rho_{3}\right)$ and, thus, for $t>T^{*}\left(\rho_{1}, 2^{-k} \rho_{1}, x_{0}\right)$, $x\left(t, x_{n}\right) \in B\left(\frac{1}{2} \rho_{2}\right)$. This contradicts the assumption that $x\left(t_{n}, x_{n}\right) \notin B\left(\rho_{2}\right)$.

Thus, $N\left(x_{0}\right)$ exists for each $x_{0} \in S\left(\rho_{1}\right)$ and the Heine-Borel theorem implies that the $T^{*}$ s have a supremum $T^{*}\left(\rho_{1}, \rho_{2}\right)$ over $S\left(\rho_{1}\right)$.

By homogeneity, $T^{*}\left(\rho_{1}, \frac{1}{2} \rho_{1}\right)=\rho_{1}{ }^{1-m} T^{*}\left(1, \frac{1}{2}\right)$. Then

$$
T^{*}\left(\rho_{1}, 2^{-k-1} \rho_{1}, x_{0}\right) \leqq T^{*}\left(\rho_{1}, 2^{-k-1} \rho_{1}\right) \leqq T^{*}\left(1, \frac{1}{2}\right) \rho_{1}{ }^{1-m} 2^{k(m-1)} D(k),
$$

where $D(k)$ is a constant between 1 and 2 independent of $\rho_{1}$. This is shown in much the same way that the earlier inequality for $t^{*}$ was derived. Thus

$$
\rho_{1} 2^{-k} \leqq b\left(T^{*}\left(\rho_{1}, 2^{-k-1} \rho_{1}, x_{0}\right)\right)^{1 /(1-m)},
$$

where $b$ is a positive constant independent of $\rho_{1}$ and $k$.

To conclude the proof, let $\left|x_{0}\right|=\rho_{1}$ and suppose that $t$ is such that for some $k>0, \rho_{1} 2^{-k-1} \leqq\left|x\left(t, x_{0}\right)\right| \leqq \rho_{1} 2^{-k}$. Then inequalities (6) and (7) above imply that

$$
a t^{1 /(1-m)} \leqq\left|x\left(t, x_{0}\right)\right| \leqq b t^{1 /(1-m)} .
$$


Here $a$ and $b$ are as defined above and we must assume that

$$
t \leqq T^{*}\left(\rho_{1}, \frac{1}{2} \rho_{1}\right)=\rho_{1}{ }^{1-m} T^{*}\left(1, \frac{1}{2}\right) .
$$

Thus, let $T=T^{*}\left(1, \frac{1}{2}\right)$.

The other lower bound is easily derived by a consideration of the differential inequality $d r / d t \geqq \alpha r^{m}$.

Remarks. Zubov [12] gave the first version of this theorem, but provided no proof. Lefschetz [8] gave a proof for the upper estimate but none for the lower estimate. Hahn [5] handled the case of a non-autonomous $A(x, t)$, homogeneous in $x$, but again only derived upper bounds.

The next theorem has to do with Lyapunov functions for system (5). The proof is due to Lefschetz [8], who clarified a somewhat obscure proof of a more general theorem of Zubov [12]. The assumptions are those made in the first paragraph of this section, and in addition assume that $m$ is an integer, and that the components of $A(x)$ are homogeneous polynomials.

THEOREM 2. There exist homogeneous polynomials $W(x)$ and $V(x), W$ negative definite and of degree $m+1, V$ positive definite and of degree 2, such that $\dot{V}(x)=W(x)$, where the derivative is computed with respect to solutions of (5).

Proof. Let $W(x)=-|X|^{m+1}, V(x)=\int_{0}^{\infty}|X(t, x)|^{m+1} d t$. To avoid confusion, $X(t, x)$ will denote the solution of (5) such that $x=X(0, x)$. That the improper integral exists is assured by the upper bound estimate of the previous theorem. That $V$ is homogeneous of degree 2 (and therefore a quadratic form since $V$ is analytic) is shown by evaluating $V(c x)$ for real $c$ :

$$
\begin{aligned}
V(c x)=\int_{0}^{\infty}|X(t, c x)|^{m+1} d t=\int_{0}^{\infty}|c|^{m+1}\left|X\left(c^{m-1} t, x\right)\right|^{m+1} d t \\
=|c|^{m+1} \int_{0}^{\infty}|X(s, x)|^{m+1} c^{1-m} d s=c^{2} \int_{0}^{\infty}|X(s, x)|^{m+1} d s=c^{2} V(x) .
\end{aligned}
$$

Here we have used the fact that $m$ is odd (a consequence of asymptotic stability) and a change of variable $s=c^{m-1}$.

In order to show that $\dot{V}(x)=W(x)$, we proceed as follows:

Therefore,

$$
V(X(t, x))=\int_{0}^{\infty}|X(\tau, X(t, x))|^{m+1} \bar{d} \tau=\int_{t}^{\infty}|X(s, x)|^{m+1} d s .
$$

$$
\frac{d V}{d t}(X(t, x))=-|X(t, x)|^{m+1}=W(X(t, x))
$$

3. Growth and decay estimates for a perturbed system. Consider the following system [i.e., (1) with slightly different conditions],

$$
\begin{aligned}
& d x / d t=A(x)+f^{*}(x, y), \\
& d y / d t=B(y)+g^{*}(x, y),
\end{aligned}
$$


where $x$ is a $k$-vector, $y$ is an $(n-k)$-vector, $A$ and $B$ are homogeneous polynomials of degree $m>1$ in the components of $x$ and $y$, respectively, and $f^{*}$ and $g^{*}$ are each $C^{1}$ and $O\left(|z|^{m+1}\right)$ in a neighbourhood of the origin, where $z=(x, y)$. It is further assumed that the stationary point at the origin of $E^{k}\left[E^{n-k}\right]$ is asymptotically stable [unstable] for the system

$$
d x / d t=A(x)[d y / d t=B(y)] .
$$

When (1) has the form of (8), we say that the stationary point at the origin is elementary, strongly non-linear, and in canonical form. These terms refer back to the case when $m=1$ and we have linear first approximation. For that case there is always a linear variable change reducing a system such as (4) (with $m=1$ ) to (8) with $A(x)=A x$ and $B(y)=B y$, where $A$ is an asymptotic stability matrix and $B$ is an asymptotic instability matrix. As mentioned in the introduction, the possibility of the reduction of (3) to (2), and thus (4) to (8), is treated in $\$ 4$. For now, we treat (8) only. Our methods resemble those of Reiziňs $[\mathbf{1 0}]$.

If $k=0$ or $n$, then one or the other of the systems of first approximation to (8) disappears. It will usually be assumed in the following that $0<k<n$, although the two cases $k=0$ and $k=n$ can be treated similarly. All later results concern the behaviour of solutions of the coupled system above near the isolated stationary point at the origin in $E^{n}$. For this reason, there will be no loss of generality if the functions $f^{*}$ and $g^{*}$ are altered outside a neighbourhood of the origin. Let $R>0$. Define $f$ and $g$ to be functions on $E^{n}$ such that $f(z) \equiv f^{*}(z)$ and $g(z) \equiv g^{*}(z)$ for all $|z| \leqq R$, while $f(z)=0$ and $g(z)=0$ for all $|z| \geqq 2 R$. For $R$ sufficiently small, such $f$ and $g$ can be found with the same differentiability properties as $f^{*}$ and $g^{*}$ and all sharing a common Lipschitz constant $L$. The Lipschitz condition on each of the four functions $f, g, f^{*}$, and $g^{*}$ is the same. We write it out only for $f$ :

$$
\left|f\left(z_{1}\right)-f\left(z_{2}\right)\right| \leqq L\left|z_{1}-z_{2}\right|^{m} .
$$

The Lipschitz inequalities for $f^{*}$ and $g^{*}$ are valid for $|z|<R$, while those for $f$ and $g$ hold for all $z$. Moreover, if $R$ is small enough, then $L$ is arbitrarily small, at least for $|z|<R$.

The two systems to be studied are the coupled system,

$$
\begin{aligned}
& d x / d t=A(x)+f(x, y), \\
& d y / d t=B(y)+g(x, y),
\end{aligned}
$$

and the uncoupled system of first approximation,

$$
\begin{aligned}
& \text { (a) } d u / d t=A(u), \\
& \text { (b) } d v / d t=B(v) .
\end{aligned}
$$

The hypotheses on the functions and the systems are those given above. In 
addition, it is assumed that $R$ is so small that (9) has no stationary point other than that at the origin. There is also no loss of generality in assuming that $d|u|^{2} / d t$ is negative definite while $d|v|^{2} / d t$ is positive definite, the derivatives being with respect to trajectories of (10) (a) and (10)(b), respectively. For Theorem 2 asserts the existence of two positive-definite quadratic forms $V_{1}(u)$ and $V_{2}(v)$ whose derivatives (with respect to trajectories of (10) (a) and $(10)(\mathrm{b})$, respectively) are $d V_{1} / d t=-|u|^{m+1}$ and $d V_{2} / d t=|v|^{m+1}$. Let $u=A^{*} u^{*}, v=B^{*} v^{*}$, reduce $V_{1}$ and $V_{2}$ to $V_{1}^{*}=\left|u^{*}\right|^{2}$ and $V_{2}^{*}=\left|v^{*}\right|^{2}$, respectively. In the new variables, $d V_{1}{ }^{*} / d t=W_{1}{ }^{*}$ and $d V_{2}{ }^{*} / d t=W_{2}{ }^{*}$, where $W_{1}{ }^{*}$ and $W_{2}{ }^{*}$ are negative and positive definite, respectively and each is homogeneous of degree $m+1$. For simplicity, it will be assumed that such a change of variables has already been made in systems (9) and (10) and the asterisks will be omitted.

How does a given trajectory of $(9)$ behave within $B(R)$, the ball of radius $R$ in $E^{n}$ centred at the origin? The next four theorems answer this question. The first theorem gives qualitative answers and, most important, implies that no trajectory can tend to the origin both as $t$ tends to $-\infty$ and as $t$ tends to $+\infty$, i.e., (9) possesses no closed loop solutions. If there were such solutions, then systems (9) and (10) could not be topologically equivalent since (10) is easily seen to have no closed loops. The remaining theorems give growth and decay estimates on solutions. These will not be exponential as in the case of linear first approximation, but of the form $a t^{1 /(1-m)}$, as might be expected from Theorem 1.

THEOREM 3. Each solution of (9) is of one of the following three types:

(a) $|z(t)| \rightarrow 0$ as $t \rightarrow \infty,|z(t)| \rightarrow \infty$ as $t$ decreases;

(b) $|z(t)| \rightarrow 0$ as $t \rightarrow-\infty,|z(t)| \rightarrow \infty$ as $t$ increases;

(c) $|z(t)| \rightarrow \infty$ as $t$ increases and as $t$ decreases.

Proof. Let $V(z)=-|x|^{2}+|y|^{2}$. Then $d V / d t=-W_{1}(x)+W_{2}(y)+h(z)$, where the derivative is with respect to trajectories of (9), $W_{1}$ and $W_{2}$ are the negative and positive definite (respectively) $m+1$ forms defined above and $h(z)$ is $O\left(|z|^{m+2}\right)$ near the origin. $R$ is assumed to be so small that $d V / d t$ is positive definite everywhere (recall that $h(z) \equiv 0$ for $|z| \geqq 2 R$ ).

$V=0$ defines the "cone" $|x|^{2}=|y|^{2}$; the cone degenerates into the stationary point if $k$ is 0 or $n$. $V=c \neq 0$ defines the "hyperboloid" $|x|^{2}=|y|^{2}-c$ or an $(n-1)$-sphere if $k=0$ or $n$. In all but the degenerate cases of the stationary point or the empty set, these hypersurfaces divide $E^{n}$. Since $d V / d t$ is positive definite and $V(0)=0$, no trajectory $z(t) \neq 0$ of $(9)$ can tend to 0 both as $t \rightarrow-\infty$ and as $t \rightarrow+\infty$. Similarly, no limit points of $z(t)$ can lie on any of the hypersurfaces $V=c$ (with the exception of the single stationary point at the origin). Thus, if $|z(t)| \rightarrow 0$ as $t$ increases (decreases), then $|z(t)| \rightarrow \infty$ as $t$ decreases (increases), and the theorem is proved. 
TheOREM 4. There exist positive constants $a$ and $b, b<a$, such that every solution $z\left(t, z_{0}\right)$ of (9) of type (a) satisfies the inequalities

$$
\frac{\left|x_{0}\right|}{\left(1+a\left|x_{0}\right|^{m-1} t\right)^{1 /(m-1)}} \leqq\left|z\left(t, z_{0}\right)\right| \leqq \frac{2\left|x_{0}\right|}{\left(1+b\left|x_{0}\right|^{m-1} t\right)^{1 /(m-1)}}
$$

for all $t \geqq 0$, where $z_{0}=\left(x_{0}, y_{0}\right)$. Moreover, for all $t,-b^{-1}\left|x_{0}\right|^{1-m}<t \leqq 0$, $z\left(t, z_{0}\right)$ satisfies the inequality

$$
\frac{\left|x_{0}\right|}{\left(1+b\left|x_{0}\right|^{m-1} t\right)^{1 /(m-1)}} \leqq\left|z\left(t, z_{0}\right)\right| \text {. }
$$

If $-a^{-1}\left|x_{\mathrm{c}}\right|^{1-m}<t \leqq 0$, then the inequality

also holds.

$$
\left|z\left(t, z_{0}\right)\right| \leqq \frac{2\left|x_{0}\right|}{\left(1+a\left|x_{0}\right|^{m-1} t\right)^{1 /(m-1)}}
$$

Proof. Let $M^{+}$be the set of solutions of (9) of type (a).

$$
V(z)=-|x|^{2}+|y|^{2}<0
$$

on every solution of $M^{+}$since $d V / d t$ is positive definite, $V(0)=0$, and every solution tends to 0 as $t \rightarrow \infty$. Thus, $|y|<|x|$ on $M^{+}$. Let

$$
a_{1}=\inf (\tilde{x} \cdot A(\tilde{x})) \leqq \sup (\tilde{x} \cdot A(\tilde{x}))=b_{1},
$$

where the infimum and supremum are taken over the sphere $|\tilde{x}|=1$ in $E^{k}$. Since $d|x|^{2} / d t$, with respect to (10)(a), is negative definite and, in fact, is $x \cdot A(x)$, it follows that $a_{1} \leqq b_{1}<0$. For $R$ sufficiently small, it then follows that

$$
2 a_{1} r^{m}<\dot{r}=r^{m}(\tilde{x} \cdot A(\tilde{x}))+\tilde{f}(z)<\frac{1}{2} b_{1} r^{m},
$$

where $r=|x|,|\tilde{x}|=1$, and $\tilde{f}(z)$ is $O\left(r^{m+1}\right)$ since $|y|<|x|$.

By comparing the three equations $\dot{r}=2 a_{1} r^{m}, \dot{r}=r^{m}(\tilde{x} \cdot A(\tilde{x}))+\tilde{f}(z)$, and $\dot{r}=\frac{1}{2} b_{1} r^{m}$, it follows that $x\left(t, z_{0}\right)$ satisfies the estimates

$$
\frac{\left|x_{0}\right|}{\left(1-2 a_{1}(m-1)\left|x_{0}\right|^{m-1} t\right)^{1 /(m-1)}} \leqq\left|x\left(t, z_{0}\right)\right| \leqq \frac{\left|x_{0}\right|}{\left(1-\frac{1}{2} b_{1}(m-1)\left|x_{0}\right|^{m-1} t\right)^{1 /(m-1)}}
$$

for $t \geqq 0$.

Noting that $|x| \leqq|z| \leqq 2|x|$ in $M^{+}$, the first inequalities of the theorem follow. The remaining inequalities follow by integrating the three equations for $\dot{r}$ backwards from $t=0$.

Theorem 5. There exist positive constants $c$ and $d, d<c$, such that every solution $z\left(t, z_{0}\right)$ of $(9)$ of type (b) satisfies the inequalities

$$
\frac{\left|y_{0}\right|}{\left(1-c\left|y_{0}\right|^{m-1} t\right)^{1 /(m-1)}} \leqq\left|z\left(t, z_{0}\right)\right| \leqq \frac{2\left|y_{0}\right|}{\left(1-d\left|y_{0}\right|^{m-1} t\right)^{1 /(m-1)}}
$$


for all $t \leqq 0$, where $z_{0}=\left(x_{0}, y_{0}\right)$. Moreover, for all $t, 0 \leqq t<d^{-1}\left|x_{0}\right|^{1-m}, z\left(t, z_{0}\right)$ satisfies the inequality

$$
\frac{\left|y_{0}\right|}{\left(1-d\left|y_{0}\right|^{m-1} t\right)^{1 /(m-1)}} \leqq\left|z\left(t, z_{0}\right)\right| .
$$

If $0 \leqq t<c^{-1}\left|x_{0}\right|^{1-m}$, then there also holds the inequality

$$
\left|z\left(t, z_{0}\right)\right| \leqq \frac{2\left|y_{0}\right|}{\left(1-c\left|y_{0}\right|^{m-1} \frac{1}{)^{1 /(m-1)}}\right.} .
$$

The proof is similar to that of the previous theorem and is omitted.

THEOREM 6. There exist positive constants $a, b, c, d$ such that every solution $z\left(t, z_{0}\right), z_{0}=\left(x_{0}, y_{0}\right),\left|x_{0}\right|=\left|y_{0}\right|$, of (9) of type (c) satisfies the inequalities

$$
\begin{gathered}
\frac{\left|x_{0}\right|}{\left(1+b\left|x_{0}\right|^{m-1} t\right)^{1 /(m-1)}} \leqq\left|z\left(t, z_{0}\right)\right| \quad \text { if }-b^{-1}\left|x_{0}\right|^{1-m}<t \leqq 0 ; \\
\left|z\left(t, z_{0}\right)\right| \leqq \frac{2\left|x_{0}\right|}{\left(1+a\left|x_{0}\right|^{m-1}\right)^{1 /(m-1)}} \quad \text { if }-a^{-1}\left|x_{0}\right|^{1-m}<t \leqq 0 ; \\
\frac{\left|x_{0}\right|}{\left(1-d\left|x_{0}\right|^{m-1} t\right)^{1 /(m-1)}} \leqq\left|z\left(t, z_{0}\right)\right| \quad \text { if } 0 \leqq t<d^{-1}\left|x_{0}\right|^{1-m} ; \\
\left|z\left(t, z_{0}\right)\right| \leqq \frac{2\left|x_{0}\right|}{\left(1-c\left|y_{0}\right|^{m-1} t\right)^{1 /(m-1)}} \quad \text { if } 0 \leqq t<c^{-1}\left|x_{0}\right|^{1-m} .
\end{gathered}
$$

Note that the condition $\left|x_{0}\right|=\left|y_{0}\right|$ is no restriction since $\dot{V}$ is positive definite.

Proof. Let $z\left(t, z_{0}\right)$ be a solution of (9) of the desired type. Then for $t<0$, $|x| \geqq|y|$ and all the analysis of the earlier theorem applies, along with the appropriate estimates. Similarly for $t \geqq 0,|x| \leqq|y|$, and the theorem is proved.

4. Canonical forms for homogeneous systems. In the previous section, we have assumed that (4) could be written in the form of system (8). Under what conditions is this possible? It is easily seen that the question is equivalent to asking for conditions under which a linear variable change reduces system (3) to system (2) where $z$ is an $n$-vector, $x$ is a $k$-vector, $y$ an $(n-k)$-vector, and $C, A$, and $B$ are homogeneous polynomials of integral degree $m \geqq 1$. In this section we shall go considerably beyond the "uncoupling" problem and shall generalize earlier work of Markus [9] to tie in the linear equivalence of homogeneous systems with isomorphism questions of non-associative algebras. Most of this section is independent of the earlier sections. Because of this, the notation is not necessarily consistent with that used earlier.

Consider the systems of autonomous, homogeneous ordinary differential equations in $n$-space,

$$
\dot{x}=f(x)
$$


and

$$
\dot{y}=g(y),
$$

where $f$ and $g$ are homogeneous polynomials of integral degree $m \geqq 1$.

System (11) is linearly equivalent to system (12) if there is a non-singular constant $n \times n$ matrix $A$ such that $A f\left(A^{-1} y\right) \equiv g(y)$. Hence, the linear map $y=A x$ maps (globally) the trajectories of (11) onto those of (12). If $f(x) \equiv B x, B$ a constant matrix, the problem of finding the "simplest" differential system linearly equivalent to $\dot{x}=B x$ is the same as the algebraic problem of finding the "simplest" matrix $A B A^{-1}$. Thus, the algebraic method of classifying matrices via Jordan normal forms (i.e., via eigenvalues and eigenspaces) yields a classification of autonomous linear differential systems up to linear equivalence. This relationship between algebraic and differential problems led Markus [9] to attempt to classify certain non-linear differential systems with strong algebraic properties by algebraic techniques. In particular, Markus considered differential systems of the form $\dot{x}=f(x)$, where $f(x)$ is a homogeneous vector polynomial of degree 2 in the components of $x$, i.e., quadratic differential systems. He showed that the problem of classifying such systems relative to linear equivalence corresponded to classifying certain, generally non-associative, algebras up to isomorphism. He then carried out completely such a classification in the case of planar quadratic systems.

Here, it is shown that there is a natural generalization of Markus' method to homogeneous polynomial systems where $f(x)$ is of arbitrary positive integral degree, not necessarily 1 or 2 . It will be seen that such things as lines of singularities, ray integrals, linear integral manifolds, and so on, have direct analogues in an algebra corresponding to the differential system. However, the algebraic restatement of the linear classification problem for the differential systems considered does not necessarily make things easier. The algebra involved will usually be non-associative, and too little is known as yet about such algebras [11]. The advantage may, in fact, be to the algebraist since more seems to be known about the differential systems than about the algebras $[\mathbf{1} ; \mathbf{4}]$.

Let $f_{i}=a_{i i_{1} \ldots i_{m}} x_{i_{1}} \ldots x_{i_{m}}$ and $g_{i}=\tilde{a}_{i i_{1} \ldots i_{m}} y_{i_{1}} \ldots y_{i_{m}}$, where $i=1, \ldots, n$; we follow the summation convention that any subscript appearing twice is summed from 1 to $n$, and the $a$ s and $\widetilde{a}$ s are constants. (11) and (12) are then homogeneous polynomial systems of degree $m$. Without loss of generality it may be assumed that $a_{i i_{1} \ldots i_{m}}$ is invariant relative to a permutation of the subscripts $i_{1}, \ldots, i_{m}$ as is $\tilde{a}_{i i_{1} \ldots i_{m}}$. The algebra $A$ associated with $(11)$ is the real algebra with basis elements $u_{1}, \ldots, u_{n}$, real scalar multiplication, algebra addition, and an $m$-nary multiplication $\mu$ defined for basis elements $u_{i_{1}}, \ldots, u_{i_{m}}$, by the formula $\mu\left(u_{i_{1}}, \ldots, u_{i_{m}}\right)=a_{i_{1} \ldots i_{m}} u_{i}$. A similar algebra $\widetilde{A}$ is associated with (12). Multiplication is commutative because of the assumption about the $a$ s. The algebra may not be associative as the following example shows: 
In the algebra associated with the planar quadratic system,

$$
\dot{x}_{1}=x_{1}{ }^{2}+2 x_{1} x_{2}+x_{2}{ }^{2}, \quad \dot{x}_{2}=x_{2}{ }^{2},
$$

the product $\mu\left(u_{1}, \mu\left(u_{2}, u_{2}\right)\right)=2 u_{1}$ while $\mu\left(\mu\left(u_{1}, u_{2}\right), u_{2}\right)=u_{1} . u$ is a nilpotent of $A$ if $\mu(u, \ldots, u)=0$; it is an idempotent if $\mu(u, \ldots, u)=\lambda u$ for some real non-zero $\lambda$. A linear subset $A^{1}$ of $A$ is a subalgebra of $A$ if $A^{1}$ is closed relative to $\mu$; it is an ideal if the $\mu$-product of any element of $A^{1}$ with any $m-1$ elements of $A$ is in $A^{1}$.

TheOREM 7. Systems (11) and (12) are linearly equivalent if and only if the associated algebras $A$ and $\widetilde{A}$ are isomorphic.

Proof. Suppose that (11) and (12) are linearly equivalent with respect to the transformation $y=B x$, where

Then,

$$
B=\left(b_{i j}\right), \quad B^{-1}=C=\left(c_{i j}\right), \quad b_{i j} c_{j k}=\delta_{i k}=c_{i j} b_{j k} .
$$

$$
\dot{y}_{i}=b_{i j} a_{j i_{1} \ldots i_{m}} c_{i_{1} j_{1}} \ldots c_{i_{m} j_{m}} y_{j_{1}} \ldots y_{j_{m}} .
$$

Therefore, $\widetilde{a}_{i j_{1} \ldots j_{m}}=b_{i j} a_{j i_{1} \ldots i_{m}} c_{i_{1} j_{1}} \ldots c_{i_{m} j_{m}}$. Now let $\phi: A \rightarrow \widetilde{A}$ be defined by $\phi u_{j}=b_{i j} \tilde{u}_{i}$, where the $u_{j}$ and $\tilde{u}_{i}$ are basis elements of $A$ and $\widetilde{A}$, respectively. $\phi$ is evidently one-to-one. In addition, $\phi \mu\left(u_{j_{1}}, \ldots, u_{j_{m}}\right)=\phi a_{i_{1} \ldots j_{m}} u_{i}=$ $b_{j i} a_{i j_{1} \ldots j_{m}} \widetilde{u}_{j}$, while $\mu\left(\phi u_{j_{1}}, \ldots, \phi u_{j_{m}}\right)=\mu\left(b_{i_{1} j_{1}} \tilde{u}_{i_{1}}, \ldots, b_{i_{m} j_{m}} \tilde{\mu}_{i_{m}}\right)=$ $b_{i_{1} j_{1}} \ldots b_{i_{m} j_{m}} \widetilde{a}_{i i_{1}} \ldots i_{i_{m}} \tilde{u}_{i}$. By the previously derived relation between the $a$ s and the $\widetilde{a}$ s, we see that $\phi \mu\left(u_{j_{1}}, \ldots, u_{j_{m}}\right)=\mu\left(\phi u_{j_{1}}, \ldots, \phi u_{j_{m}}\right)$ and $A$ is isomorphic to $\widetilde{A}$.

On the other hand, if $A$ is isomorphic to $\widetilde{A}$ via the isomorphism $\phi$ defined by $\phi u_{j}=b_{i j} \tilde{u}_{i}$ on the basis elements, then $y=\left(b_{i j}\right) x$ defines an invertible map of (11) onto (12), as is seen by reversing the above arguments.

Since nilpotents, idempotents, subalgebras, and ideals are isomorphic invariants, there is no loss of generality in assuming each of these in a "favourable" position relative to a basis. This will be done repeatedly in the proofs of the following theorems.

THEOREM 8. $\alpha_{i} u_{i}$ is a nilpotent of $A$ if and only if $\lambda\left(\alpha_{1}, \ldots, \alpha_{n}\right)$ is a line of critical points of (11).

Proof. $u_{1}$ is a nilpotent of $A$ if and only if $\mu\left(u_{1}, \ldots, u_{1}\right)=a_{i 1 \ldots 1} u_{i}=0$, which holds if and only if $a_{i 1 \ldots 1}=0$ for each $i$. This, in turn, is true if and only if $\lambda(1,0, \ldots, 0)$ is a line of critical points of $(11)$.

THEOREM 9. $\alpha_{i} u_{i}$ is an idempotent of $A$ if and only if the two rays $\lambda\left(\alpha_{1}, \ldots, \alpha_{n}\right)$ corresponding to $\lambda<0$ and $\lambda>0$ are non-critical integral rays of (11).

Proof. $u_{1}$ is an idempotent of $A$ if and only if

$$
\mu\left(u_{1}, \ldots, u_{1}\right)=a_{i 1 \ldots 1} u_{i}=\lambda u_{1}
$$

for some non-zero scalar $\lambda$, which is equivalent to the assertion that $a_{11 \ldots 1} \neq 0$, 
$a_{i 1 \ldots 1}=0, i>1$. This is true if and only if $\beta(t)(1,0, \ldots, 0)$, where $\beta(t)=$ $a_{11 \ldots 1}\left[1+(1-m) a_{1}^{m-1} \ldots 1 t\right]^{1 /(1-m)}$, defines a non-critical ray trajectory of (11).

Theorem 10. If $n$ is odd, $A$ has at least one nilpotent or idempotent and (11) has at least one line of critical points or pair of opposed integral rays.

Proof. Because of the homogeneity, the vector field defined by (11) can be projected along radial rays onto the unit sphere $S^{n-1}$ centred at the origin. This yields a continuous tangent field on $S^{n-1}$. Under this projection a ray which is either a ray of critical points or a non-critical integral ray corresponds to a critical point of the projected field on $S^{n-1}$, and conversely. If $n$ is odd, any field on $S^{n-1}$ is known to have at least one critical point. By the symmetry of the systems considered here, critical points on $S^{n-1}$ occur in antipodal pairs, and the theorem is proved.

Theorem 11. The elements $v_{j}=\alpha_{i j} u_{i}, i=1, \ldots, r$, of $A$ span an $r$-dimensional subalgebra of $A$ if and only if the hyperplane spanned by the vectors $\left(\alpha_{1 j}, \ldots, \alpha_{n j}\right), j=1, \ldots, r$, is an integral manifold of (11).

Proof. Let $\alpha_{i j}=\delta_{i j}$. Then $u_{1}, \ldots, u_{r}$ span an $r$-dimensional subalgebra if and only if $\mu\left(u_{j_{1}}, \ldots, u_{j_{m}}\right)=a_{i j_{1} \ldots j_{m}} u_{i}$ is in the span of $u_{1}, \ldots, u_{r}$ whenever $\left\{u_{j_{1}}, \ldots, u_{j_{m}}\right\} \subset\left\{u_{1}, \ldots, u_{r}\right\}$. This is true if and only if $a_{i j_{1} \ldots j_{m}}=0$ for all $i>r, j_{k} \leqq r, k=1, \ldots, m$, which is equivalent to the assertion that the subset of $E^{n}$ for which $x_{r+1}=\ldots=x_{n}=0$, i.e., the hyperplane generated by the $x_{1}, \ldots, x_{r}$ axes, is an in tegral manifold of (11).

Theorem 12. The elements $v_{j}=\alpha_{i j} u_{i}, j=1, \ldots, r$, of $A$ span an $r$-dimensional ideal of $A$ if and only if (11) is linearly equivalent to a system (12) where $\tilde{a}_{i j_{1} \ldots j_{m}}=0$ for $i>r$ and $j_{k} \leqq r$ for at least one $k$.

Proof. Let $\alpha_{i j}=\delta_{i j}$. Then $u_{1}, \ldots, u_{r}$ span an $r$-dimensional ideal if and only if $\mu\left(u_{j_{1}}, \ldots, u_{j_{m}}\right)=a_{i_{1} \ldots j_{m}} u_{i}$ is in the span of $u_{1}, \ldots, u_{r}$ whenever at least one of the $u_{j_{i}}$ s belongs to the set $\left\{u_{1}, \ldots, u_{r}\right\}$. This is equivalent to the assertion that $a_{i j_{1} \ldots i m}=0$ if $i>r$ and $i_{k} \leqq r$ for at least one $k$.

Corollary 1. $A$ is the direct sum of $s$ ideals if and only if (11) is linearly equivalent to a direct product of $s$ homogeneous polynomial systems of degree $m$.

Now, Markus [9] classified all planar quadratic differential systems up to linear equivalence by actually calculating (using idempotents and nilpotents) all possible two-dimensional algebras $A$. To do this even for the algebra defined by a cubic differential system would be a formidable task. In any event, linear equivalence is much stronger than is usually needed for qualitative studies of differential systems. It might be noted that the idempotents are the "eigenvalues" for homogeneous systems, but that, contrary to the linear case, the idempotents do not determine the system up to linear equivalence. 
Corollary 2. System (11) is linearly equivalent to the following system

$$
\begin{aligned}
& \dot{y}_{1}=g_{1}\left(y_{1}\right), \\
& \dot{y}_{2}=g_{2}\left(y_{2}\right),
\end{aligned}
$$

where $y_{1}$ is a k-vector, $y_{2}$ an $(n-k)$-vector, $g_{1}$ and $g_{2}$ homogeneous of degree $m$, if and only if $A$ is the direct sum of two ideals.

Remark. This corollary implies, then, that (4) can be written in the form of (8) if and only if the algebra defined by the homogeneous first approximation of (4) has the property mentioned in the corollary.

\section{REFERENCES}

1. C. Coleman, Systems of differential equations without linear terms, Internat. Sympos. Nonlinear differential equations and nonlinear mechanics, pp. 445-453 (Academic, New York, 1963).

2. W. A. Coppel, Stability and asymptotic behavior of differential equations (Heath, Boston, 1965).

3. D. M. Grobman, Topological classification of neighborhoods of a singularity in n-space, Mat. Sb. (N. S.) 56 (98) (1962), 77-94. (Russian)

4. W. Hahn, Stability of motion (Springer, New York, 1967).

5. — Über Differentialgleichungen erster Ordnung mit homogenen rechten Seiten, Z. Angew. Math. Mech. 46 (1966), 357-361.

6. P. Hartman, Ordinary differential equations (Wiley, New York, 1964).

7. - On the local linearization of differential equations, Proc. Amer. Math. Soc. 14 (1963), $568-573$.

8. S. Lefschetz, The critical case in differential equations, Bol. Soc. Mat. Mexicanna (2) 6 (1961), 5-18.

9. L. Markus, Quadratic differential equations and non-associative algebras; Contributions to the theory of nonlinear oscillations, Vol. V, pp. 185-213 (Princeton Univ. Press, Princeton, N. J., 1960).

10. L. E. Reizinsš, Local topological equivalence of systems of differential equations, Differencial'nye Uravnenija 4 (1968), 199-214. (Russian)

11. R. D. Schafer, An introduction to nonassociative algebras (Academic Press, New York, 1966).

12. V. I. Zubov, Methods of A. M. Lyapunov and their application, Technical Report AEC-tr.4439, Nuclear Science Abstracts (32435) 15 (1961), 4183 (translated from Metody A. M. Lyapunova i. ikh Primenenie; publication of the Publishing House of Leningrad University, 1957).

Harvey Mudd College, Claremont, California 